\title{
Revisión teórica y aplicación práctica de las ciencias del espacio para reducir el consumo de combustibles en cohetes y vehículos espaciales*
}

| Fecha de recibido: 5 de marzo del 2020 | Fecha de aprobación: 29 de abril del 2020 |

\author{
TC. Guillermo Alberto \\ Poveda Zamora \\ Magíster en Ingeniería con énfasis \\ en Ingeniería Aeroespacial \\ Investigador de la Escuela de Postgrados \\ de la Fuerza Aérea Colombiana. \\ Grupo de Investigación CIPAER \\ Rol de investigador: intelectual, \\ experimental, comunicativo \\ https://orcid.org/0000-0002-3358-102X \\ $\triangle$ guillermo.poveda@epfac.edu.co
}

* Artículo de investigación derivado del proyecto Diseño y creación de la Maestría en Ingeniería Aeroespacial de la Fuerza Aérea Colombiana.

Cómo citar este artículo: Poveda Zamora, G. A. Revisión teórica y aplicación práctica de las ciencias del espacio para reducir el consumo de combustibles en cohetes y vehículos espaciales. Revista Ciencia y Poder Aéreo, 15(1), 152-160. https://doi.org/10.18667/cienciaypoderaereo.657 


\section{Revisión teórica y aplicación práctica de las ciencias del espacio para reducir el consumo de combustibles en cohetes y vehículos espaciales}

Resumen: El propósito de este artículo es hacer un acercamiento a los fundamentos básicos de las ciencias del espacio en torno a la geometría, velocidad de la Tierra, mecánica celeste y el aprovechamiento natural de las ventajas del planeta con autosostenibilidad y rentabilidad del negocio espacial. El documento aborda temas como la posición de los lanzamientos espaciales y costos globales de poner cargas útiles en el espacio, con una mirada de las ciencias del espacio, los combustibles, la energía y los vehículos espaciales extra pesados. En el desarrollo del texto se utilizaron algunas técnicas de investigación cualitativa, para describir el posible efecto económico de la cuantificación del ahorro de los lanzamientos aeroespaciales en las regiones y la industria aeroespacial. Se analiza además la ventaja que ofrecen las características físicas naturales de la posición espacial de Colombia y doce países más, ubicados en diferentes continentes, los cuales, por su ubicación geográfica, tienen territorio sobre la latitud $0^{\circ}$ en el globo terráqueo. Esta información permitirá que el lector se acerque de manera sencilla a un área de conocimiento específica, con la intención de promover el saber espacial con aspectos propios de la ciencia que servirán como herramienta para la apropiación de conceptos, análisis contextual y estímulo de la comprensión crítica y reflexiva. Esto servirá como fuente dinamizadora en la investigación de un campo poco estudiado hasta hoy, pero con inmensas posibilidades de exploración y múltiples perspectivas de beneficio social.

Palabras clave: Economía y sostenibilidad; línea del Ecuador; vehículos espaciales; velocidad de rotación.

Abstract: This paper approaches the basic foundations of Space Sciences around geometry, earth speed, celestial mechanics, and the use of the advantages offered by our planet for the self-sustainability and profitability of the space industry. The study addresses issues such as the position of space launches and the general costs of putting payloads into space, with a particular focus on Space Science, fuel, energy, and super-heavy spacecraft. In the development of the text some qualitative research techniques were used to describe the possible economic effects of quantifying the savings of space launches, examining the benefits offered by the natural physical characteristics of the spatial position of Colombia and 12 more countries located on different continents, which, due to their geographical location, count on lands at zero latitude on the globe. This information will allow the reader to easily approach a specific area of knowledge with the intention of promoting spatial knowledge through science as a tool for understanding new concepts, favoring contextual analysis and stimulating critical and reflective thinking as a dynamic source of research in a field little studied until now, but with immense possibilities for exploration and multiple benefits for the society.

Keywords: Economy and Sustainability; Equator; Spacecraft; Rotation Speed.

Resumo: 0 objetivo deste artigo é abordar os fundamentos básicos das ciências do espaço em torno da geometria, velocidade da Terra, a mecânica celeste e o aproveitamento natural das vantagens do planeta em referência à auto-sustentabilidade e rentabilidade do negócio espacial. O escrito aborda questões como a posição dos lançamentos espaciais e os custos globais de colocar cargas úteis no espaço, com uma perspectiva das ciências do espaço, os combustíveis, a energia e os veículos espaciais extrapesados. No desenvolvimento do texto foram utilizadas algumas técnicas de pesquisa qualitativas, para descrever o possível impacto econômico que poderia causar nas regiões e na indústria aeroespacial, realizar uma aproximação que quantifique a economia dos lançamentos espaciais, analisando a vantagem oferecida pelas características físicas naturais da posição espacial da Colômbia e mais 12 países localizados em continentes diferentes, que por causa da sua localização geográfica, têm acima de zero de latitude no globo terrestre. Esta informação permitirá ao leitor se aproximar de um modo simples a uma área de conhecimento específica, com a intenção de promover o saber espacial com aspectos próprios da ciência que servirão como recurso para a apropriação de conceitos, análise contextual e estímulo da compreensão crítica e reflexiva, como princípio dinâmico de pesquisa em um campo ainda pouco estudado, mas com imensas possibilidades de estudo e múltiplas perspectivas de benefício social.

Palavras-chave: Economia e sustentabilidade; Linha do Equador; Veículos espaciais e velocidade rotacional. 


\section{Introducción}

Considerando la creciente demanda de obtención de recursos naturales vitales como el agua, el oxígeno, la flora y la fauna, garantes de la sostenibilidad de los pueblos, también cobra gran importancia la necesidad de ahorrar en el consumo de combustibles fósiles, los cuales contaminan y afectan importantes reservas naturales del planeta y, por ende, los recursos naturales y la biodiversidad. Es claro que Colombia no es ajena a este daño ambiental, ya que tiene una de las regiones más ricas en reservas minerales, además de tener el bosque tropical más extenso del planeta Tierra (World Wild Fund for Nature [wWF], 2015).

La ascendente carestía de alimentos, del líquido vital y las declaraciones de la Organización de las Naciones Unidas (ONU), quienes prevén que para 2050 "una cuarta parte de la población mundial sobrevivirá en países con una falta crónica de agua limpia" (Palou, 2017), nos hacen reflexionar sobre la importancia de disminuir el consumo de combustibles líquidos y sólidos, acción que a la vez permitirá proteger los recursos naturales que poseen los seres humanos y demás especies habitantes del planeta (ONU, 2017). Se espera que para el 2050 la población mundial sea de 8900 millones de habitantes (De la Varga, 2005), lo que significa que habrá 2225 millones de personas sin acceso al agua potable. Esta proyección debe ser tenida en cuenta por los países para desarrollar todos los esfuerzos posibles en materia de gestión eficiente de sus recursos. En este caso particular, el artículo se direcciona hacia los planes o proyectos en la creciente industria aeroespacial de explotación de recursos desde el espacio con el fin de disminuir el consumo de combustibles líquidos y sólidos en cohetes y vehículos espaciales.

Con los datos señalados hasta este punto, surge la necesidad de buscar elementos basados en la ciencia, la tecnología, los procedimientos, la revisión de las teorías y leyes de la física y la matemática, que permitan explotar las ventajas naturales propias de nuestro planeta para reducir la contaminación generada por cohetes y vehículos espaciales en sus recorridos hacia el espacio. Esta contaminación ha sido estudiada desde el Programa de las Naciones Unidas para el Medio Ambiente (PNUMA) y, además, cuenta con apoyo y financiamiento de la Administración Nacional de la Aeronáutica y del Espacio (NASA), la Comisión Espacial Europea, la Organización Mundial de la Salud (OMS) y la National Oceanic and Atmospheric Administration (NOAA) (2018). Dicho lo anterior, y a la par de los nuevos desarrollos tecnológicos, se busca presentar soluciones con resultados más eficientes en el transporte de personas, material, equipo y diferentes tipos de suministros para la explotación del espacio, así como la proyección del ser humano fuera de nuestro sistema solar (Schelling, 1980), paso inevitable en la evolución de la sociedad y conciencia humana (White, 1998).

Dentro de la misión constitucional y doctrina propia del uso del poder aéreo de la Fuerza Aérea Colombiana (FAC), está "[...] contribuir a los fines del Estado" (FAC, 2019), lo cual supone una apuesta permanente en el desarrollo tecnológico y el mejoramiento de procesos y estrategias para garantizar el cumplimiento de la función constitucional plasmada en el artículo 217 de la Constitución Política de Colombia (1991). Según esta premisa, y acatando los objetivos en materia de incursionar en la carrera espacial y la protección del medio ambiente, surge una gran incógnita ¿la posición geográfica de Colombia puede brindar ventajas naturales para el lanzamiento de vehículos espaciales y hacer más eficiente el uso de combustibles líquidos y sólidos?

\section{Método}

Para dar respuesta al interrogante, se aplicó un método exploratorio del conocimiento disponible, que tiene como objetivo aumentar el grado de entendimiento de una actividad relativamente desconocida en Colombia, la cual puede ser materializada en una investigación completa, profunda y con la rigurosidad científica en el desarrollo de las ciencias del espacio. Así mismo, es un método mixto de tipo descriptivo, en la medida 
que detalla analíticamente el comportamiento de una variable asociada a la forma y la gravedad de la Tierra (García, 2010); a las leyes de Newton, en una estrecha relación con la mecánica celeste; las leyes de Kepler, y el uso de combustibles líquidos y sólidos para la generación de velocidad requerida para que los vehículos espaciales abandonen la Tierra y posicionen cargas útiles en órbitas alrededor del planeta.

A su vez, se aplican herramientas de análisis matemático en las leyes de la física para demostrar cómo las ventajas naturales que ofrece el planeta Tierra pueden permitir un uso eficiente de los combustibles para el lanzamiento de vehículos espaciales, en contraposición a los costos de poner cargas útiles en el espacio.

\section{Resultados y análisis}

Definir la teoría usada en el presente artículo supone tener presente la forma de la Tierra, ya que en ella se construye el concepto matemático que demuestra la ventaja de la posición natural de la línea del Ecuador en el globo terráqueo para el lanzamiento de vehículos espaciales y cohetes (Poveda \& Álvarez, 2020).

La fuerza de gravedad es perpendicular a la superficie de la Tierra en cualquiera de sus puntos, como se puede observar en la figura 1. Las componentes de la línea de color rojo representan una tangente sobre cualquier punto de la superficie y, por otra parte, en la línea de color azul, se puede evidenciar cómo la gravedad resulta ser un componente vertical de fuerza hacia el centro del planeta.

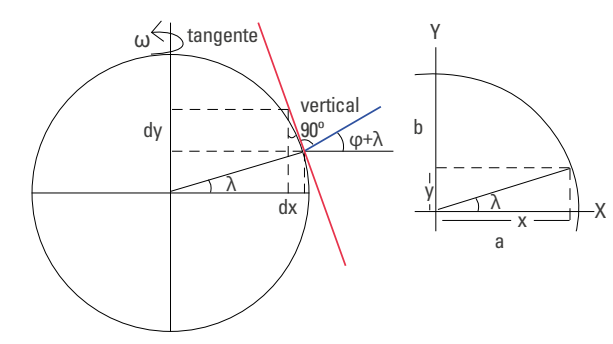

Figura 1. Forma de la Tierra y componente de fuerza de la gravedad Fuente: García (2010).
Como la Tierra es un geoide achatado en el centro, y no una esfera perfecta, se tienen diferentes mediciones físicas dependiendo del punto de la Tierra en donde se tomen las medidas (como la gravedad, o el radio medido desde el centro de la Tierra hasta algún punto en la superficie, entre otras medidas; véase a figura 1).

Considerando que la Tierra no es una circunferencia, sino que se parece a una elipse (Piskunov \& Medkov, 1983), se parte de la siguiente ecuación:

$$
\frac{x^{2}}{a^{2}}+\frac{y^{2}}{b^{2}}=1
$$

Al hacer los cálculos de la elipse, en concordancia con lo planteado en la figura 1 , se obtienen los valores que han sido presentados por The International Astronomical Union (IAU) (1984), así:

$$
\left\{\begin{array}{l}
a=\text { radio ecuatorial }=6378,14 \mathrm{~km} \\
b=\text { radio en los polos }=6356,75 \mathrm{~km}
\end{array}\right.
$$

Respecto a la velocidad de rotación de la Tierra, la velocidad angular experimentada por los cuerpos en su superficie cambia significativamente dependiendo del lugar o posición donde ellos se encuentren. Presentan una velocidad angular máxima en el radio donde la Tierra es mayor, es decir, sobre la línea del Ecuador, y mínima en los polos (Poveda \&Álvarez, 2020).

Atendiendo el análisis del movimiento angular propuesto por Zare (1988), sabiendo que una vuelta del planeta es $2 \pi$ radianes, y considerando que $f$ es frecuencia de rotación y $T$ el periodo, se plantea que:

$$
\begin{gathered}
\frac{v}{r}=\frac{2 \pi}{T}=\omega=2 \pi f \\
v=\omega r
\end{gathered}
$$

En la matemática y física relacionada con vehículos espaciales, cuerpos celestes y todo tipo de masa en interrelación con el planeta Tierra, se considera que el movimiento genera cambios de sus velocidades y energías (Poveda, 2016). 
La energía es representada de la siguiente forma:

$$
\begin{aligned}
& E_{\text {cinética }}=1 / 2 m v^{2} \\
& E_{\text {potencial }}=\frac{G M r}{(1.6)} \\
& E_{\text {total }}=1 / 2 m v^{2}-\frac{G M}{r}
\end{aligned}
$$

De las expresiones descritas anteriormente, se define $m$ como la masa del vehículo espacial (Universidad Politécnica de Madrid [UPM], 2013), G como constante de gravitación universal (De Orus et al., 2007) y $M$ como masa de la Tierra (Neff et al., 1995):

$$
\begin{gathered}
G=6,671 \times 10^{-11}\left[\frac{m^{3}}{\left(K g * S_{e g}\right)}\right] \\
M=5.9790 \times 10^{24} \mathrm{~kg}
\end{gathered}
$$

La relación entre velocidad objetivo y combustible requerido, de acuerdo a la École Polytechnique Fédérale de Lausanne (EPFL) (2018), y aplicando la fórmula de Tsiolkosvky (Lavavasseur, 2004), se define de manera general en la figura 2, que expone el comportamiento del consumo de combustible en vehículos espaciales:

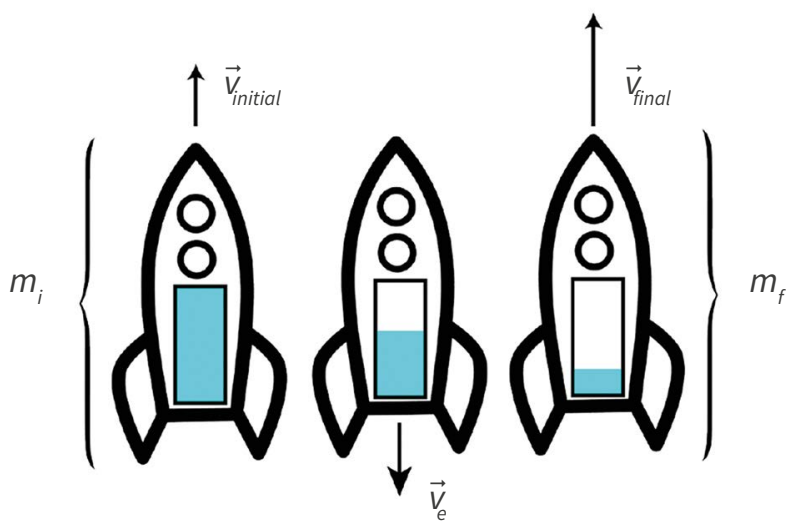

Figura 2. Consumo de combustible Fuente: EPFL (2018).

A continuación, se relaciona la velocidad inicial $\vec{v}_{\text {initial }}$, la velocidad de empuje $\vec{v}_{e}$, la velocidad final $\vec{v}_{\text {final }}$ y las dos masas, tanto la inicial como la final $m_{i}$ y $m_{f}$, y a la fórmula de Tsiolkosvky se le incorpora el concepto de impulso específico $I_{s p}$ y fuerza de la gravedad $g$, como se muestra en la siguiente expresión matemática (EPFL, 2018):

$\Delta V=g * l_{s p} * \log _{e}\left(\frac{m_{i}}{m_{f}}\right) \Longrightarrow\left\{\begin{array}{l}m_{p}=m_{i}\left[1-e^{\left.\left(-\frac{-\Delta V}{g * s_{s p}}\right)\right]}\right. \\ m_{p}=m_{f}\left[e\left(-\frac{-\Delta V}{g * s_{s p}}\right)-1\right]\end{array}\right.$

La velocidad de empuje ideal es aproximadamente de $4,5 \mathrm{~km} / \mathrm{s}$ (EPFL, 2018), con lo cual el vehículo logrará una velocidad de escape entre $5 \mathrm{~km} / \mathrm{s}$ y $10 \mathrm{~km} / \mathrm{s}$, consumiendo el $90 \%$ del combustible a bordo del vehículo, como se describe en la figura 3.

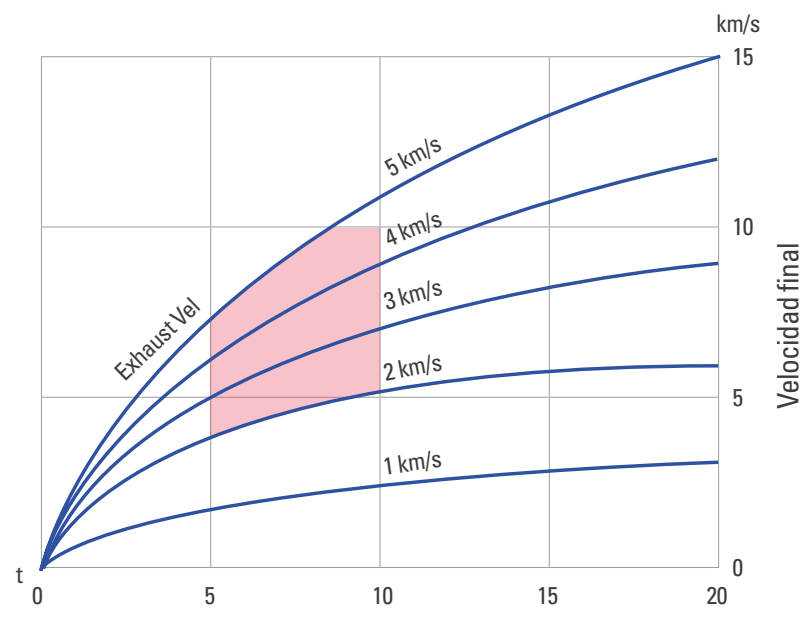

Figura 3. Impulso específico y velocidad del vehículo Fuente: Poveda \& Álvarez (2020, p. 243).

Si bien el artículo busca analizar el consumo de combustible, se tiene en cuenta el impulso específico $l_{s p}$ que se relaciona según el tipo de combustible empleado, dividiéndose en tres grandes grupos:

1. Oxígeno-Hidrógeno líquido $I_{s p}=450 \mathrm{~s}$ igual a $4,5 \mathrm{~km} / \mathrm{s}$ de $\overrightarrow{v_{e}}$.

2. Nitrógeno tetroxidado-UDMH $l_{s p}=280$ a $300 \mathrm{~s}$ igual a 2,8 a $3,0 \mathrm{~km} / \mathrm{s}$ de $\vec{v}$.

3. Monopropelentes-Hidracina $I_{s p}=200$ a $250 \mathrm{~s}$ igual a 2 a $2,5 \mathrm{~km} / \mathrm{s}$ de $\vec{v}$. 


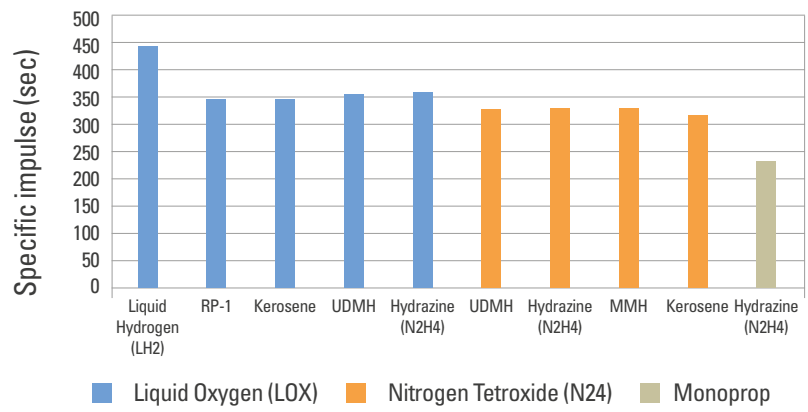

Figura 4. Impulso específico por tipo de combustible Fuente: Graham (2019) y EPFL (2018).

Ahora bien, el consumo de combustible en vehículos espaciales posicionados sobre la línea del Ecuador está relacionado con el tipo de vehículo y con la velocidad de escape a desarrollar, para de esta manera restar la velocidad de rotación, que será una velocidad extra aplicada al vehículo por estar en latitud cero.

Para hallar esa velocidad, se requiere el valor del radio de la Tierra según la latitud. Para la latitud cero será de 6378,14 km, como se muestra en la figura 5:

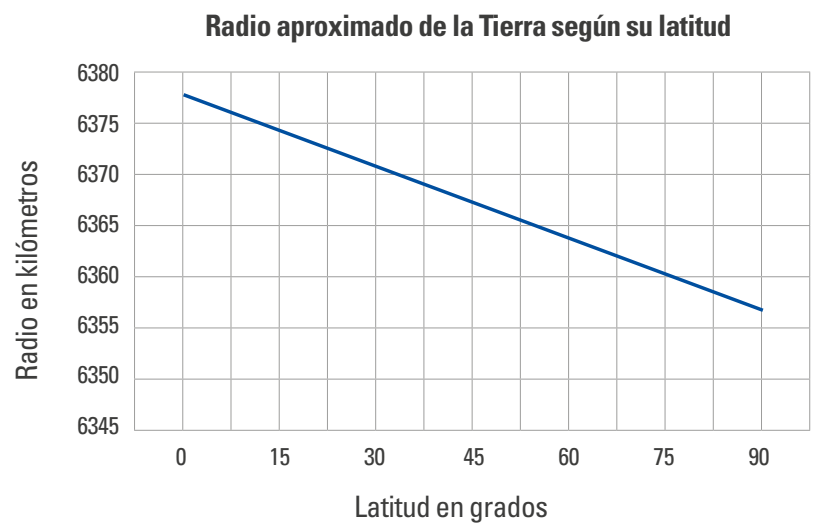

Figura 5. Radio de la Tierra vs. Latitud

Fuente: elaboración propia, información de Poveda \& Álvarez (2020, p. 246).

Asociando las ecuaciones (1.3) y (1.4) con la latitud, y tomando al radio de la Tierra según su latitud medida, se hallan las velocidades angulares requeridas para los cálculos de combustible, como se aprecia en los resultados en la figura 6 :

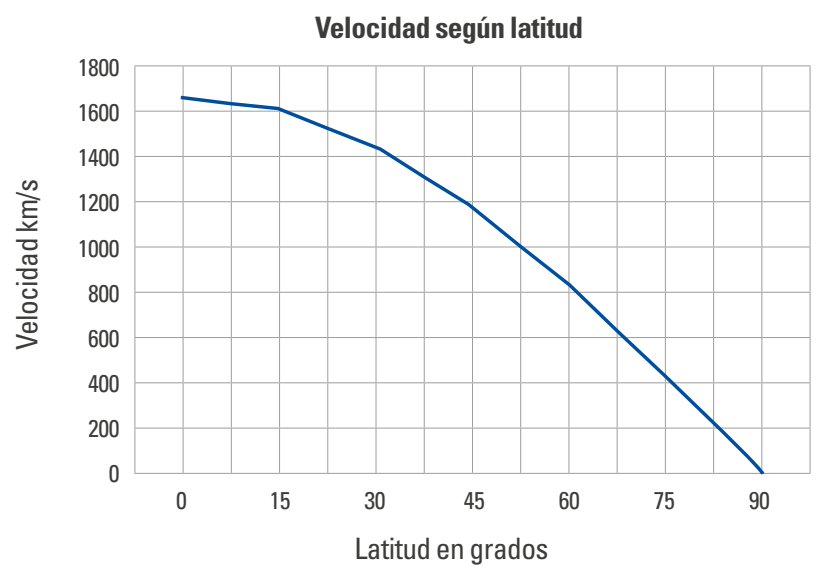

Figura 6. Curva de velocidad vs. Latitud

Fuente: elaboración propia, información de Poveda \& Alvarez (2020, p. 247) y Universidad de Sevilla $(2002$, p. 1).

Para desglosar de manera práctica el ejemplo de un vehículo espacial de combustible líquido propulsor (que puede ser de combustible sólido o líquido [Godínez, 2012]), se toma el vehículo tipo Starship de SpaceX, que usa combustible de tipo LOx y RP-1, tal como puede observarse en la figura 7:

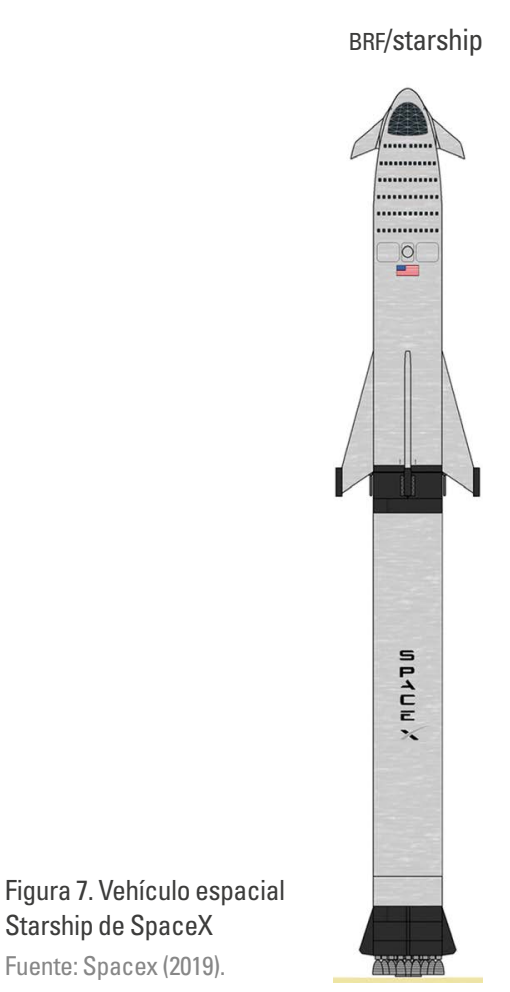


Los aspectos técnicos genéricos para este ejercicio, usando el vehículo espacial Delta Iv Heavy serán:

- Apogeo: $300 \mathrm{~km}$
- Masa total del vehículo: $5000000 \mathrm{~kg}$ total del vehículo
- Masa de la carga útil: $100000 \mathrm{~kg}$
- Velocidad: $8 \mathrm{~km} / \mathrm{s}$

Usando las expresiones desde la (1.5) hasta la (1.9), se obtienen los resultados de energía para el Starship de SpaceX sobre la línea del Ecuador (latitud cero $0^{\circ}$ ). Se cuenta con un gasto menor de energía como se observa en la tabla 1.

Tabla 1.

Gasto de energía según la latitud

\begin{tabular}{|c|c|c|c|}
\hline $\begin{array}{l}\text { Latitud } \\
\text { en grados }\end{array}$ & $\begin{array}{l}\text { Radio } \\
\text { en km }\end{array}$ & $\begin{array}{c}\text { Energía } \\
\text { potencial }\end{array}$ & $\begin{array}{l}\text { Energía } \\
\text { cinética }\end{array}$ \\
\hline 0 & $6.678,14$ & $57,17 \times 10^{6} \mathrm{~J}$ & \multirow{7}{*}{$16 \times 10^{13} \mathrm{~J}$} \\
\hline 15 & $6.674,57$ & $57,20 \times 10^{6} \mathrm{~J}$ & \\
\hline 30 & $6.671,01$ & $57,23 \times 10^{6} \mathrm{~J}$ & \\
\hline 45 & $6.667,44$ & $57,25 \times 10^{6} \mathrm{~J}$ & \\
\hline 60 & $6.663,88$ & $57,28 \times 10^{6} \mathrm{~J}$ & \\
\hline 75 & $6.660,31$ & $57,31 \times 10^{6} \mathrm{~J}$ & \\
\hline 90 & $6.656,75$ & $57,34 \times 10^{6} \mathrm{~J}$ & \\
\hline
\end{tabular}

Fuente: elaboración propia.

Al analizar la tabla 1, se muestra que en latitud $30^{\circ}$ norte, cercana al Kennedy Space Center, existe un gasto mayor de energía que en la línea del Ecuador.

Dicho lo anterior, en la tabla 2 se observan las velocidades requeridas por el vehículo Starship de SpaceX, si se consideran las latitudes de lanzamiento $30^{\circ} \mathrm{y}$ sobre la línea del Ecuador (latitud cero $0^{\circ}$ ).

Tabla 2.

Velocidades de escape del vehículo espacial en latitud $0^{\circ}, 30^{\circ}$ y $90^{\circ}$

\begin{tabular}{c|c|c|c|c|}
$\begin{array}{c}\text { Velocidad } \\
\text { de escape } \\
\text { en } \mathbf{~ k m / s}\end{array}$ & $\begin{array}{c}\text { Latitud en } \\
\text { grados }\end{array}$ & $\begin{array}{c}\text { Velocidad } \\
\text { de rotación } \\
\mathbf{k m} / \mathbf{s}\end{array}$ & $\begin{array}{c}\text { Velocidad } \\
\text { requerida } \\
\text { en } \mathbf{~ m m} / \mathbf{s}\end{array}$ & $\begin{array}{c}\text { Velocidad } \\
\text { requerida en } \\
\mathbf{k m} / \mathbf{h}\end{array}$ \\
\hline \multirow{2}{*}{8} & 0 & 0,456417715 & 7,50 & 27.174 \\
\cline { 2 - 5 } & 30 & 0,381407955 & 7,62 & 27.400 \\
\cline { 2 - 5 } & 90 & $1,51132 \mathrm{E}-07$ & 8,00 & 28.845 \\
\hline
\end{tabular}

Fuente: elaboración propia.

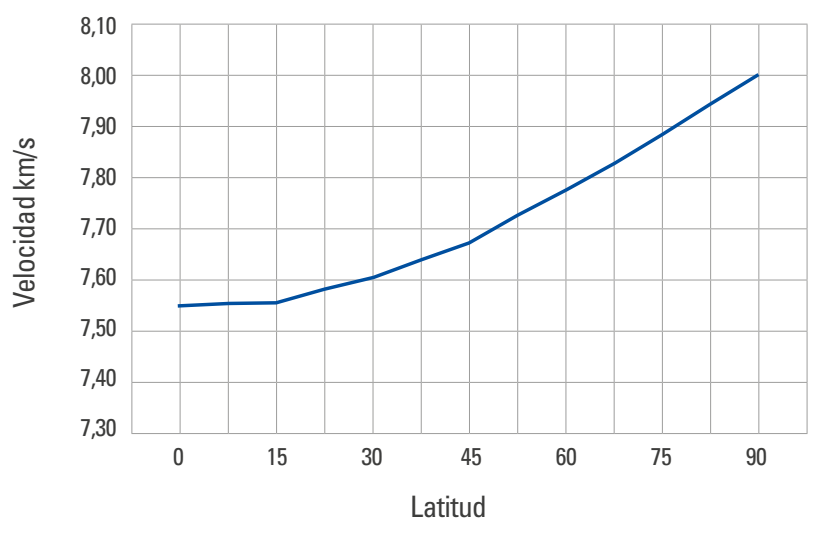

Figura 8. Velocidad de escape requerida vs. Latitud Fuente: Poveda \& Álvarez (2020, p. 247).

En relación con lo anterior, se indica que el lanzador economiza velocidad en la línea del Ecuador, pues la ubicación hace que cierta velocidad sea añadida de manera natural por la rotación de la Tierra, que es de $225,5 \mathrm{~km} / \mathrm{h}$, lo que, en un vehículo de una masa de $5 \mathrm{mi}$ llones de kilos, representa un ahorro significativo de energía para alcanzar la velocidad de escape.

Retornando al cohete Starship de SpaceX y sus características (véase en la figura 9), se debe tener en cuenta que consume la siguiente cantidad aproximada de combustible líquido:

\section{Masa de combustible Lox: 2040 toneladas Masa de combustible RP-1: 951,62 toneladas}

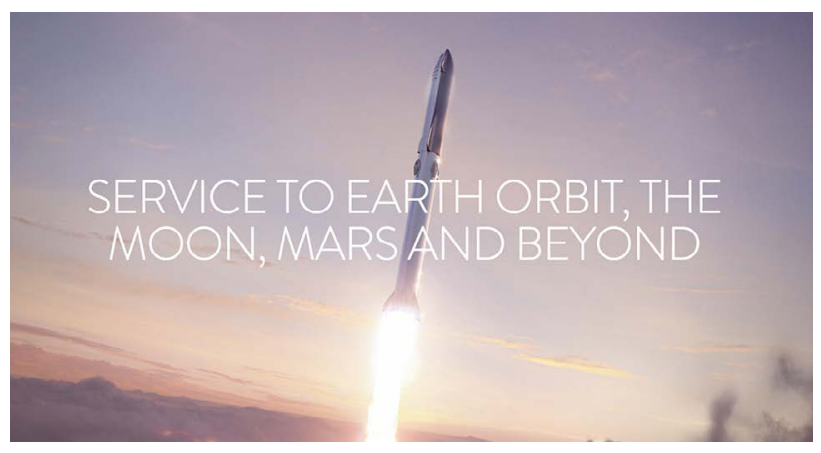

Figura 9. Cohete Starship de SpaceX Fuente: SpaceX (2020). 
Las características especiales de este cohete están asociadas a su gran tamaño y capacidad de transporte interplanetario con la que fue concebido desde su diseño inicial.

Considerando que el Oxígeno-Hidrógeno y líquido Lox tiene un $l_{s p}=450 \mathrm{~s} \circ 4,5 \mathrm{~km} / \mathrm{s}$, se usa la expresión (1.10) con las siguientes variables:

- $m$ : masa de combustible inicial.

- $m$ : masa de combustible final.

- $\Delta V$ : variación de velocidad según la latitud que fue hallada en $\mathrm{km} / \mathrm{s}$.

- La velocidad final se halla al consumir el $90 \%$ del combustible.

$$
\left\{\begin{array}{l}
m_{p}=m_{i}\left[1-e^{\left(-\frac{-\Delta V}{g * I_{s p}}\right)}\right] \\
m_{p}=m_{f}\left[e^{\left(-\frac{-\Delta v}{g * I_{s p}}\right)-1}\right]
\end{array}\right. \text { Ecuación general }
$$

Se realizan los cálculos para las dos cantidades de combustible, Lox y RP-1, en las dos latitudes de análisis, la línea del Ecuador o latitud cero $0^{\circ}$ y la latitud $30^{\circ}$. Se obtienen los siguientes resultados:

Tabla 3.

Consumos de combustible

\begin{tabular}{|c|c|c|c|}
\hline $\begin{array}{c}\text { Latitud en } \\
\text { grados }\end{array}$ & $\begin{array}{c}\text { Velocidad } \\
\text { requerida en } \mathbf{k m} / \mathbf{s}\end{array}$ & $\begin{array}{c}\text { Consumo de } \\
\text { LoX en } \mathbf{~ k g}\end{array}$ & $\begin{array}{c}\text { Consumo de } \\
\text { RP-1 en kg }\end{array}$ \\
\hline 0 & 7,50 & 1.667 .580 & 777.936 \\
\hline 30 & 7,62 & 1.678 .389 & 782.936 \\
\hline Diferencia en kg & & $\mathbf{1 0 . 8 0 9}$ & $\mathbf{5 . 0 0 0}$ \\
\hline
\end{tabular}

Fuente: elaboración propia.

La tabla 3 permite identificar una diferencia de $10.809 \mathrm{~kg}$ en combustible Lox y de 5.000 kg en el RP-1. Si se suman las masas de diferencia entre Lox y RP-1 se tiene una diferencia total de $15.809 \mathrm{~kg}$.

De esta manera, se puede inferir que al lanzar desde la línea del Ecuador un cohete Starship de SpaceX, el ahorro en masa sumado de Lox y RP-1 es de $15.809 \mathrm{~kg}$ de combustible, que se puede aprovechar en más carga útil y que representa un ahorro económico significativo de combustible.
El costo promedio del LOx es USD 7,50 por $\mathrm{kg}$ (Wade, s.f) y el de RP-1 es USD 5,30 por kg (Quimi-Net, 2016). Así pues, el ahorro en combustible Lox y RP-1 al lanzar desde la línea del Ecuador será:

- $\quad$ LOX USD 81.067,50

- $\quad$ RP-1 USD 26.500,39

El ahorro en costo del combustible será aproximadamente de USD 107.567,89.

\section{Conclusiones}

Como resultado del análisis y sus variables, se tienen las siguientes conclusiones:

1. Aplicar las teorías físicas y matemáticas al lanzar cohetes o vehículos espaciales permite comprobar que se requiere menor cantidad de combustible al lanzar desde la línea del Ecuador, aprovechando la velocidad de rotación de nuestro planeta.

2. Pese a que las teorías y leyes de la física que explican cómo funciona nuestro planeta son tan antiguas, se demuestra con estos cálculos su aplicación en el mundo actual, presentando resultados que favorecen la resolución de problemáticas actuales, tales como la preservación de nuestra atmósfera y la sostenibilidad de la carrera espacial en la humanidad.

3. Los cohetes y vehículos espaciales en su ascenso hacia el espacio exterior y las fronteras ultraterrestres liberan gases contaminantes y partículas perjudiciales para la atmósfera, de manera que reducir las emisiones de combustible en $15.809 \mathrm{~kg}$ o $15 \mathrm{t}$ en promedio para vehículos como el Starship de SpaceX representa una importante iniciativa en la preservación y cuidado de la atmósfera, sin restringir el avance del ser humano al espacio. Cada kilo de emisiones cuenta en la sumatoria global de esfuerzos por preservar nuestro planeta. 
4. Si $15.809 \mathrm{~kg}$ de ahorro en combustible se remplazaran por carga paga, en términos económicos el ahorro sería significativo. Estos resultados invitan a hacer estudios más profundos en temas de inversión y retorno para la construcción de cosmódromos o estaciones de lanzamiento sobre la línea del Ecuador que favorezcan la industria aeroespacial con la posible baja de los costos de poner cargas útiles en el espacio.

\section{Referencias bibliográficas}

Colombia. (1991). Constitución Política de Colombia. Legis.

De la Varga, J. (2005, 5 de diciembre). La escasez de agua, origen de conflictos bélicos para el siglo xxı. Forum $L i$ bertas. http://www.forumlibertas.com/la-escasez-deagua-origen-de-conflictos-belicos-para-el-siglo-xxi/

De Orus, J., Català, M., \& De Murga, J. (2007). Astronomía esférica y mecánica celeste. Universitat Barcelona.

École Polytechnique Fédérale de Lausanne. (2018). Space Mission Design and Operations. https://edu.epfl.ch/coursebook/fr/space-mission-design-and-operations-EE-585

Fuerza Aérea Colombiana. (2019, 22 de julio). Disposición 026. https://www.fac.mil.co/transparencia-y-acceso-infor macion-publica/3-estructura-organica-y-talento-huma no/mision-vision

García, A. (2010, diciembre). La forma de la Tierra. Física con ordenador. http://www.sc.ehu.es/sbweb/fisica/celeste/ forma/forma.htm

Godínez, A. (2012). La defensa contra misiles balísticos. Posible participación de las fragatas F-100. Instituto Español de Estudios Estratégicos.

Graham, W. (2019, 19 de enero). ULA Delta IV-Heavy Launches NROL-71 Following Lengthy Delay. NASA. https://www. nasaspaceflight.com/2019/01/ula-delta-iv-heavy-setto-launch-nrol-71/

International Astronomical Union. (1984). xIIth General Assembly. https://www.iau.org/static/resolutions/IAU1964_ French.pdf

Lavavasseur, D. (2004). The Tsiolkovsky Formula. Astrosurf. http://www.astrosurf.com/levavasseur/tsiolkovsky/tsiol kovsky_fichiers/Tsiolkovsky.pdf

National Oceanicand Atmospheric Administration (NOAA) (2018, octubre). wMo/UnEP Scientific Assessments of Ozone
Depletion. https://www.esrl.noaa.gov/csd/assessments /ozone/

Neff, R., Zitewitz, P., \& Davids, M. (1995). Physics, Principles and Problems. Glencoe/McGraw Hill.

Palou, N. (2017, 13 de junio). La guerra del agua, un conflicto no tan lejano. La vanguardia. https://www.lavanguardia. com/vivo/ecologia/20170613/423359841404/agua-es casez-conflictos-recursos-problema-onu.html

Piskunov, N., \& Medkov, K. (1983). Cálculo diferencial e integral. Editorial Mir.

Poveda, G. (2016). Propuesta de órbita geoestacionaria para el satélite artificial FACSAT01 simulación [Tesis de maestría]. Universidad del Valle.

Poveda, G., \& Álvarez, C. (2020). Colombia y la órbita geoestacionaria: un vínculo geoestratégico inalienable. En C. G. Álvarez (ed.), El espacio exterior: una oportunidad infinita para Colombia. El cielo no es el límite: el futuro estelar de Colombia, 2 (pp. 162-263). Escuela Superior de Guerra "General Rafael Reyes Prieto".

Quimi-Net. (2016, 13 de Enero). Precios de oxígeno (líquido). https://www.quiminet.com/productos/oxigeno-liquido-238320545/precios.htm

Schelling, T. (1980). The strategy of conflict. Harvard University Press. SpaceX. (Enero del 2020). Service to Earth Orbit, the Moon, Mars and Beyond. https://www.spacex.com/starship

SpaceX [r/SpaceXLounge]. (2019, 26 de enero). Starship vs. New Glenn (Size Comparison) [Entrada foro online]. Reddit. https://www.reddit.com/r/SpaceXLounge/comments/ ajyxx8/starship_vs_new_glenn_size_comparison/

Universidad de Sevilla. (2002). Velocidad de un punto en la superficie de la Tierra (G.I.A.). La Place. http://laplace. us.es/wiki/index.php/Velocidad_de_un_punto_en_la_ superficie_de_la_Tierra_(G.I.A.)

Universidad Politécnica de Madrid. (2013). Ecuaciones de dimensión. http://ocw.upm.es/pluginfile.php/381/mod_ label/intro/Teor_060CW.pdf

Wade, M. (s.f.). LH2. Astronautix. http://www.astronautix. com/l/lh2.html

White, F. (1998). The Overview Effect: Space Exploration and Human Evolution. American Institute of Aeronautic and Astronautic.

World Wild Fund for Nature. (2015, 5 de marzo). La Amazonía: una región de records. https://wwf.panda.org/es/que_ hacemos/sitios_prioritarios/amazonia/la_amazonia_ naturaleza/

Zare, R. (1988). Angular Momentum: Understanding Spatial Aspects in Chemistry and Physics. John Wiley \& Sons. 